\title{
Safety of concurrent administration of dexrazoxane and doxorubicin in the canine cancer patient*
}

\section{Keywords}

chemotherapy, dexrazoxane, doxorubicin, toxicity
Correspondence address: B. E. Kitchell Department of Small Animal Clinical Sciences, Michigan State University Veterinary Teaching Hospital

East Lansing,

$\mathrm{MI}$, USA

e-mail:

kitchell@cvm.msu.edu

\author{
W. M. FitzPatrick, N. G. Dervisis and B. E. Kitchell \\ Department of Small Animal Clinical Sciences, College of Veterinary Medicine, Michigan State University, East \\ Lansing, MI, USA
}

\begin{abstract}
Doxorubicin may cause a rare but serious cardiotoxicity. Dexrazoxane is a cardioprotectant drug used to reduce the risk of cardiotoxicity in human patients. In this study, 25 tumour-bearing dogs were treated with concurrent doxorubicin and dexrazoxane. The total number of doses of dexrazoxane given was 54 (range 1-5 doses per dog, median 2 doses). Five dogs received more than $165 \mathrm{mg} \mathrm{m}^{2}$ cumulative doxorubicin dose before starting dexrazoxane. Haematologic, gastrointestinal and cardiovascular toxicities were considered tolerable. The combination of doxorubicin with dexrazoxane was well tolerated with minimal side-effects in this patient cohort. Future studies are required to evaluate potential cardioprotective effects of dexrazoxane given concurrently with doxorubicin.
\end{abstract}

\section{Introduction}

Doxorubicin is an anthracycline antibiotic agent that acts as a topoisomerase II inhibitor. This classical cytotoxic agent has been a mainstay of medical oncology for more than 40 years and is used for the treatment of a wide variety of human malignancies. In canine cancer patients, doxorubicin is used to treat a similar array of tumours including lymphomas, soft-tissue sarcomas, osteosarcomas and histiocytic diseases, among other indications. Repeated administration of doxorubicin can result in a dose-dependent potentially irreversible cardiomyopathy. ${ }^{1}$ Doxorubicin's chemotherapeutic effects are mediated by several complex biomolecular events. ${ }^{2}$ Suggested mechanisms of cytotoxicity include intercalation into DNA leading to inhibition of macromolecule synthesis; generation of reactive oxygen species (ROS) leading to

* Portions of this work were presented in abstract form at the 28th Annual Conference of the Veterinary Cancer Society, October 19, 2008, Seattle, WA.
DNA damage or lipid peroxidation; DNA binding, alkylation or cross-linking; interference with DNA unwinding or DNA strand separation and helicase activity; direct membrane effects; initiation of DNA damage via inhibition of topoisomerase II and apoptosis in response to topoisomerase II inhibition. $^{3,4}$ Drugs that inhibit topoisomerase II can be classified as being either catalytic inhibitors or DNA cleavage drugs, depending on where the site of enzyme action occurs in the catalytic cycle. ${ }^{5,6}$ Doxorubicin's DNA cleavage-enhancing effect stabilizes the DNA-enzyme complex in its cleaved conformation, which inhibits resealing and leads to DNA double-strand breaks and cell death. , $^{3,7-10}$

In human patients, retrospective studies have identified risk factors for increased anthracyclineinduced chronic cardiomyopathy. ${ }^{11,12}$ Examples of risk factors include age greater than 70 years, ionizing radiation to the chest wall, pre-existing cardiac disease and co-administration of other cytotoxic agents such as cyclophosphamide and paclitaxel. ${ }^{11}$ Pre-existing cardiac risk factors for developing 
cardiotoxicity include active congestive heart failure (CHF), history of myocardial infarction within the preceding year, hypertension, aortic stenosis, diabetes mellitus and previous anthracycline administration. ${ }^{11}$ Children especially appear to be at increased risk for doxorubicin cardiotoxicity. ${ }^{11}$ The progression of cardiomyopathy in human patients is variable. ${ }^{11}$ Reduction in left ventricular ejection fraction and persistent signs of CHF are possible. ${ }^{13}$ Compensation and cardiac remodelling are believed to occur in these cases to some extent. ${ }^{14}$ Risk factors for doxorubicin cardiotoxicity in the dog have not been fully elucidated. One factor that has been shown to be a risk for the development of $\mathrm{CHF}$ in the dog is body weight. ${ }^{15}$ However, history of pre-existent cardiac disease, cumulative doxorubicin dose exceeding $240 \mathrm{mg} \mathrm{m}^{2}$, as well as other aetiologies may confer risk similar to that reported for human cancer patients. ${ }^{16}$

At the molecular level, doxorubicin-induced cardiotoxicity is variably attributed to several different mechanisms. The primary mechanism for cardiotoxicity is thought to derive from the generation of ROS by doxorubicin. ${ }^{11}$ This ROS production is catalysed by the complexing of doxorubicin with iron. The quinone groups on the $\mathrm{B}$ ring of the anthracene structure are reduced, leading to the production of a semiquinone radical that is further reduced to the alcohol, doxorubicinol. Interaction of doxorubicinol with oxygen yields oxygen free radicals. The semiquinone reacts with hydrogen peroxide producing a hydroxyl radical in the tissues. These subsequent reactions can occur in the presence or absence of iron. ${ }^{17,18}$ Free radicals can result in damage at different intracellular sites such as the nuclear envelope, cell membrane, mitochondria, DNA and sarcoplasmic reticulum. ${ }^{18,19}$ In the cardiac myocyte in particular, damage to the sarcoplasmic reticulum results in a decrease in bound calcium. ${ }^{11}$ Contractility is decreased by the effects of low bound calcium on the actin-myosin complex. ${ }^{11}$ Free $\mathrm{Ca}^{++}$can also activate proteases within the myocardium, consequently damaging myofibrils. ${ }^{11}$ Another proposed mechanism of myocardial damage associated with doxorubicin is thought to be altered levels of cellular free radical scavenging enzymes including superoxide dismutase (SOD), catalase and glutathione peroxidase. ${ }^{11}$
After doxorubicin exposure, myocardial levels of glutathione peroxidase decrease while SOD level is unaffected. ${ }^{11}$ It should be noted that in some mammalian systems, cardiac myocytes have low catalase levels compared with the amount of this enzyme seen in other organs..$^{20}$ Cardioprotective agents such as dexrazoxane mitigate cardiac myocyte damage caused by doxorubicin but not damage induced by the related anthracenedione derivative mitoxantrone. This finding suggests possible unexplained mechanisms for chemotherapy-induced damage induced by anthracyclines. ${ }^{21}$

More recently, it has been postulated that the mitochondria is the primary target of doxorubicin-induced oxidative stress. Doxorubicin has been shown to induce heart-specific mutations and quantitative defects in mitochondrial DNA (mtDNA). ${ }^{1,22,23}$ This results in a cardiac impairment of mtDNA-encoded respiratory chain subunits and dysfunction of the respiratory chain, promoting ROS release. Therefore, it is believed that somatically acquired mtDNA lesions play an essential role in the doxorubicin's 'dose memory', leading to the clinical onset of cardiomyopathy with lifelong cumulative doxorubicin dosing. ${ }^{22}$

In addition, oxidative stress is a primary mechanism of doxorubicin cardiotoxicity resulting in increased ROS liberation, ${ }^{2,18,24,25}$ lipid peroxidation $^{25-27}$ and decreased levels of antioxidants and sulfhydryl groups. ${ }^{28-30}$ There are many other potential contributory mechanisms that have been postulated for doxorubicin-induced cardiomyopathy. Table 1 illustrates the many proposed mechanisms of doxorubicin cardiotoxicity, which are reviewed by Takemura and Fujiwara. ${ }^{2}$

Dexrazoxane is a bisdioxopiperazine used to protect against doxorubicin-induced cardiotoxicity. ${ }^{5,9,10}$ Dexrazoxane has been approved by the Food and Drug Administration to decrease myocardial toxicity of doxorubicin in human patients with metastatic breast cancer receiving a cumulative doxorubicin dose greater than $300 \mathrm{mg} \mathrm{m}^{2}$ and was more recently approved for treatment of anthracycline extravasation. ${ }^{31}$ Initially, dexrazoxane was evaluated for anti-cancer properties, but the drug was found to have limited activity as a cytotoxic agent. The cardioprotective mechanism was traditionally believed to be related to the iron-chelating 
Table 1. Proposed mechanisms of doxorubicin-induced cardiotoxicity

Generation of reactive oxygen species

Damage to nuclear envelope, cell membrane, mitochondria, DNA and sarcoplasmic reticulum

Altered levels of free radical scavengers

Inhibition of nucleic acid and protein synthesis

Release of vasoactive amines (histamines, catecholamines and prostaglandins)

Alteration of adrenergic function and adenylate cyclase activity

Mitochondrial abnormalities

Lysosomal changes

Modification of sarcolemmal $\mathrm{Ca}^{2+}$ transport

Alteration of $\mathrm{Na}^{+}-\mathrm{K}^{+}$ATPase and $\mathrm{Ca}^{++}$-ATPase activities

Imbalance in myocardial electrolytes

Impairment of the membrane binding, assembly and enzymatic activity of mitochondrial creatinine kinase

Induction of nitric oxide synthase, leading to nitric oxide and peroxynitrite production and nitration/inactivation of myofibrillar creatine kinase and/or metalloproteinases

Reduction of expression of cardiac-specific genes, possibly by affecting expression and function of dox-sensitive transcriptional regulatory proteins

Induction of apoptosis in vascular cells and cardiac myocytes, demonstrated by caspase activation and internucleosomal DNA degradation

properties of the ring-opened metabolite of dexrazoxane, ADR-925. ${ }^{32,33}$ The ring-closed form of the parent compound is an effective topoisomerase II catalytic inhibitor ${ }^{34-36}$ that stabilizes the DNA-enzyme complex in a 'closed clamp' conformation. This results in the enzyme being less sensitive to cleavage enhancers. ${ }^{36}$ As a result, dexrazoxane inhibits enzymatic activity without causing DNA strand breaks. ${ }^{5}$ More recently, there have been a preponderance of studies suggesting other mechanisms of cardioprotection of dexrazoxane. Examples include blocking apoptosis in ventricular myocytes ${ }^{37}$ and identifying that daunorubicin-induced apoptosis may involve downstream products of superoxide which can be blocked by chelators and SOD. ${ }^{38}$ Additionally, selective cardiotoxicity may be demonstrated in rat embryonic cardiomyocytes but not adult cardiomyocytes. ${ }^{39}$ This has been illustrated by showing that the apoptotic pathway is more active in immature cardiac cells than in adult cardiac cells. Furthermore, the contribution of the cytochrome c-Apaf 1-caspase-9 pathway to doxorubicin cardiotoxicity may be limited in the adult myocardium. This may in part explain the increased incidence of developing dilative cardiomyopathy and chronic heart problems in children exposed to anthracyclines. It is noteworthy to consider that the same may be true in our canine patients. In addition, work by Lyu et al. ${ }^{40}$ revealed that dexrazoxane can antagonize the formation of Top $2 \alpha$ and Top $2 \beta$ cleavage complexes. Dexrazoxane can also induce specific degradation of the Top $2 \beta$ isozyme which is present in the hearts of adult mice, while Top $2 \alpha$ is not. Consequently, Lyu et al. postulate two possible mechanisms that explain the cardioprotective effects of dexrazoxane as regards doxorubicininduced DNA damage. These proposed mechanisms are direct interference with formation of the Top 2 cleavage complexes and Top $2 \beta$ downregulation via proteasomal degradation.

Dexrazoxane was evaluated in preclinical dog studies that demonstrated its safety when administered to normal $\operatorname{dogs}^{33}$ and in conjunction with doxorubicin. ${ }^{41}$ To date there are no published studies evaluating the safety or cardioprotective effects of dexrazoxane in clinical canine cancer patients that have received doxorubicin.

In this report, we describe our experience with the use of dexrazoxane in dogs treated with doxorubicin (dox/dex) for a variety of neoplastic diseases. Our hypothesis is that dexrazoxane can be safely administered to canine cancer patients in conjunction with doxorubicin.

\section{Materials and methods}

For inclusion in this study, dogs had to meet at least one of the following criteria: pre-existing diagnosed clinical heart disease; onset of impaired systolic 
function during the course of doxorubicin therapy; cumulative dose of doxorubicin of $180 \mathrm{mg} \mathrm{m}^{2}$ or cardiac disease as determined by echocardiographic evaluation. Dogs were not included specifically for subclinical valvular insufficiency in the absence of one of the aforementioned cardiac concerns.

Evaluated parameters included the following: fractional shortening (FS), fractional area (FA), left ventricular area during systole and diastole, left ventricular internal dimension during systole and diastole. Echocardiographic criteria for defining significant cardiac impairment (systolic dysfunction) included having a FA less than $40 \%$ and/or FS less than 25\%. For standardization purposes, the serial echocardiography data were re-evaluated by a single cardiologist. The baseline echocardiogram was defined as the study conducted before the first dose of dexrazoxane, regardless of prior doxorubicin exposure. The comparator echocardiogram was the final echocardiogram performed for each patient. Statistical evaluation of cardiac function status between these time points was analysed by the paired Student's $t$-test using standard software (PASW-Statistics 17, 2009; SPSS, Chicago, IL, USA).

\section{Drug administration}

Dexrazoxane was administered for $5-10 \mathrm{~min}$ as an intravenous (IV) infusion to patients at a level of 10 times the administered milligram dose of doxorubicin, $10 \mathrm{~min}$ before the chemotherapy agent was given. Doxorubicin was administered IV in $0.9 \% \mathrm{NaCl}$ for $25 \mathrm{~min}$ at a dose of $1 \mathrm{mg} / \mathrm{kg}$ for patients weighing less than $10 \mathrm{~kg}$ and $30 \mathrm{mg} \mathrm{m}^{2}$ for patients weighing more than $10 \mathrm{~kg}$.

Patients were serially evaluated by means of physical examination, echocardiography, electrocardiography, complete blood counts, serum biochemistry panels and urinalyses. Neoplastic disease status was re-assessed by means of caliper measurements of enlarged peripheral lymph nodes, thoracic radiographs and abdominal ultrasonography. Additional clinical data collected included follow-up for survival duration, cause of death and determination of any cardiac, haematologic and gastrointestinal toxicities [assessed by Veterinary Cooperative Oncology Group (VCOG) toxicity scores]. ${ }^{37,42}$

\section{Results}

Twenty-five client-owned tumour-bearing dogs were included in this retrospective study. Dogs received dexrazoxane concurrently with doxorubicin during the study period, which extended from January 2003 to December 2008 at the Veterinary Teaching Hospital of Michigan State University (MSU). Patient demographics included 9 neutered males, 3 intact males, 12 spayed females and 1 intact female. Breeds represented included five Golden Retrievers, three mixed breed dogs, three Rottweilers, two Labrador Retrievers, two Shi Tzus, two Boxers and one each of the following breeds: German Shepherd, Havenese, English Terrier, Borzoi, Chesapeake Bay Retriever, English Springer Spaniel, Shetland Sheepdog and Siberian Husky. Ages of the dogs in this study population ranged from 4 to 12 years, with a mean age of 8 years. Weights ranged from 4.9 to $50 \mathrm{~kg}$; doxorubicin dose was calculated based on the estimation of lean body weight. Four of the dogs had pre-existing heart disease and one had uncontrolled hypertension at the time of inclusion in the study. Two Golden Retrievers had mitral regurgitation coupled with decreased systolic function on echocardiogram, one Boxer had tricuspid valvular insufficiency and frequent unifocal ventricular premature contractions (VPCs) and one Shi Tzu had both mitral and tricuspid insufficiency with left atrial enlargement. The cancers treated in these 25 dogs included 15 lymphomas, 7 osteosarcomas and 1 each of fibrosarcoma, haemangiosarcoma and carcinoma.

The total number of doses of dexrazoxane given was 54 (range $1-5$ per dog; median 2). The cumulative doxorubicin dose received before the first dose of dexrazoxane was administered ranged from 0 to $180 \mathrm{mg} \mathrm{m}^{2}$. Five dogs received more than $165 \mathrm{mg} \mathrm{m}^{2}$ cumulative doxorubicin dose before starting dexrazoxane. The median cumulative doxorubicin dose administered with dexrazoxane was $105 \mathrm{mg} / \mathrm{m}^{2}$ (range $30-270 \mathrm{mg} / \mathrm{m}^{2}$ ). One dog received a single dose of dexrazoxane to counteract a suspected minimal volume of doxorubicin extravasation; this patient was excluded from the cardiac analysis.

Haematologic, gastrointestinal and cardiovascular toxicities were assessed using the published 
VCOG criteria. $^{42}$ There were 35 episodes of haematologic toxicity noted during the 54 doses of dox/dex administered in the course of this study. Aneamias ranged from Grade 1 (13 of 54, 24\%) to Grade 2 (7 of 54, 12.9\%), while neutropenia was observed to encompass Grade 1 (8 of 54, 14.8\%), Grade 2 ( 1 of $54,1.8 \%$ ), Grade 3 ( 1 of $54,1.8 \%$ ) and Grade 4 ( 2 of $54,3.7 \%$ ). Thrombocytopenias were seen at the toxicity level of Grade 1 (7 of 54, 12.9\%) and Grade 2 ( 1 of 54, 1.8\%). Gastrointestinal toxicity events were observed in 10 patients through the course of our study. Gastrointestinal toxicities were reported to be emesis of Grades 1 ( 6 of 54, 11.1\%) and 2 ( 3 of 54,5.5\%), and Grade 1 diarrhoea (9 of $54,16.6 \%)$.

Of the seven dogs with serial echocardiographic studies available for review, no statistically significant changes were noted for any of the aforementioned cardiac parameters. There was no statistically significant evidence of decline in systolic function after administration of dox/dex.

Treatment was stopped for three dogs because of the attending oncologists' assessment of progressive heart disease during the course of the study. One Shi Tzu with severe mitral regurgitation, mild tricuspid regurgitation and increased left atrial size went on to develop clinical signs of CHF. At the time $\mathrm{CHF}$ was suspected, this patient was discovered to have aspiration pneumonia. The dog subsequently succumbed to sepsis and multi-organ failure. This aspiration event occurred 3 weeks after the eight dose of doxorubicin (one dexrazoxane dose was coadministered with the final dose of doxorubicin). One Boxer with moderate tricuspid regurgitation but without heart failure had occasional VPCs. The attending oncology clinician elected to discontinue doxorubicin because of the enlargement of the right atrium on thoracic radiographs, but progressive heart disease was not corroborated by echocardiographic assessment. The final dog was an English Terrier that had moderate mitral regurgitation at initiation of therapy, with subsequent decrement in systolic function (FS decreased from 20.2 to $18.8 \%$ ). The clinician elected to discontinue treatment primarily because of owner's concern for gastrointestinal toxicity and also concern for cardiac toxicity based on a cardiologist's assessment of increased risk for cardiotoxicity with additional doxorubicin administration. For two of these three dogs for whom serial echocardiographic evaluation was performed, no statistical change in echocardiographic values was detected. The remaining 22 dogs discontinued the dox/dex protocol because of tumour progression. No deaths specifically attributable to heart disease were observed in this patient cohort.

\section{Discussion}

To the authors' knowledge, this is the first report evaluating the safety of dexrazoxane in the clinical setting in the canine patient. In this admittedly limited study, dexrazoxane appeared to be well tolerated. We found the rates of gastrointestinal and haematologic toxicity of dox/dex in the present study to be comparable to those reported in the historical veterinary literature for doxorubicin alone. In one study by Mutsaers et al., ${ }^{43}$ the incidence of severe gastrointestinal toxicities from single-agent doxorubicin used to treat canine lymphoma was $17 \%$. Postorino et al. ${ }^{15}$ found the incidence of moderate to severe gastrointestinal and haematologic toxicities to be 24 and 11\%, respectively, in dogs treated for lymphosarcoma. In a study of 133 dogs receiving doxorubicin as a 1-h infusion to treat a variety of different tumours, neutropenia occurred in 24 dogs (18\%), gastrointestinal toxicity in 21 dogs $(15.7 \%)$, sepsis in 3 dogs $(2.2 \%)$, thrombocytopenia in 5 dogs (3.7\%) and concurrent gastrointestinal toxicity and neutropenia in 7 dogs (5.2\%). ${ }^{44}$ A dose-intensified doxorubicin protocol for haemangiosarcoma in 20 dogs found that 5 dogs had a Grade 1 neutropenia, 3 dogs had a Grade 2 neutropenia and 1 dog had a Grade 3 neutropenia. In this study of dose-intense doxorubicin reported by Sorenmo et al.$^{45}$, five dogs required dose reductions because of neutropenia or gastroenteritis, while only one required a delay in treatment because of prolonged neutropenia.

In the present study, haematologic and gastrointestinal toxicities were mostly mild. Anaemia, neutropenia and thrombocytopenia were mostly classified as Grade 1 toxicity. Gastrointestinal toxicities occurred in 10 patients; however, six episodes of Grade 1 and three episodes of Grade 2 emesis were recorded and nine of the diarrhoea episodes noted were Grade 1. Thus, it appears that the addition 
of dexrazoxane to doxorubicin in treatment of tumour-bearing dogs did not appreciably increase the incidence of common cytotoxic adverse effects. It should be noted that toxic effects may be influenced by dosing on lean body mass rather than total body weight basis. In human patients, evaluation of alternate size descriptors for dose calculation in obese patients has limitations. ${ }^{46}$ The same may be true for canine patients and prospective trials are needed to address dosing chemotherapeutic drugs based on body weight. Future prospective studies need to be performed to compare incidence of gastrointestinal or other toxicities in dogs that received $1 \mathrm{mg} / \mathrm{kg}$ and $30 \mathrm{mg} \mathrm{m}^{2}$ of doxorubicin to identify any inherent sensitivities based on body weight.

There are several limitations of this study. Chief among these are the small sample size and retrospective nature of the study. In addition, only 14 dogs had serial echocardiograms to evaluate for potential alterations of systolic function during the course of therapy. Of these 14 dogs, only 7 had echocardiographic data that could be reevaluated by a single cardiologist to limit interindividual variability in calculation of the different echocardiographic parameters. This unfortunate circumstance was attributable to data retrieval issues of the original echocardiographic unit when a different manufacturer's equipment was adopted at MSU. Thus, while numerical measured values were retrievable from the medical records of these patients, the actual echocardiogram images could not be recovered for re-assessment. One of the dogs (English Terrier) with lost original echocardiographic images developed clinical signs of systolic dysfunction and evidence of mild progressive heart disease (FS decreased from 20.2 to $18.8 \%$ ). The second dog (Boxer) had improved systolic function echocardiographically, but right atrial enlargement on thoracic radiographs.

No information regarding efficacy of cardioprotection can be gleaned from this work. In human patients, the true incidence of cardiotoxicity caused by doxorubicin is very low $(\sim 4 \%)$ for patients who received $500-550 \mathrm{mg} \mathrm{m}^{2} .{ }^{47}$ Previous studies in veterinary medicine have shown an incidence of doxorubicin-induced cardiotoxicity ranging from 2 to $29.8 \% .^{15,44,45,48-50}$ The $29.8 \%$ cardiotoxicity rate was reported in a cohort of dogs treated with concurrent doxorubicin and whole-body hyperthermia. ${ }^{50}$ The incidence of cardiotoxicity in that study was similar between dogs that received whole-body hyperthermia and those that did not, suggesting that whole-body hyperthermia alone was not a significant contributor. In that study onset of previously undetected arrhythmia was considered a toxicity, which may have contributed to the high reported incidence. Furthermore, dogs had echocardiograms with every treatment, which may have lead to a lower threshold of detection of cardiac changes than in the present work. ${ }^{50}$

The low rate of possible cardiac toxicity noted here is consistent with past reports, and in fact is perhaps lower as the threshold for declaring cardiac change was low in our study. Variance in measurements and definitions of cardiotoxicity thresholds between studies make direct comparison difficult if not impossible. It is unclear whether the administration of dexrazoxane had any role in modulating the cardiac status of any of the cases in this series, where therapy was discontinued because of clinician's concerns for progressive heart disease. It is unfortunate that serial echocardiographic data were not available on all dogs in this retrospective study. Echocardiography is clearly helpful, along with other markers of myocardial injury such as serum levels of cardiac troponin, although one might argue that echocardiography alone has insufficient sensitivity to detect early myocardial injury.

The present study was underpowered to evaluate any cardioprotective effect in dogs without preexisting heart disease. For dogs treated with doxorubicin, power analysis indicates that to detect a $50 \%$ reduction in the incidence of cardiotoxicity in tumour-bearing dogs with normal baseline cardiac function (from 7.5 to $3.75 \%{ }^{48}$ incidence), with alpha of $P<0.05$ and power of $80 \%, 589$ dogs would be required for each of two groups, comprising the dox/dex treatment arm and a dox-alone control arm. This analysis is based on the assumption that all dogs would receive $180 \mathrm{mg} \mathrm{m}^{2}$ cumulative doxorubicin dose, with no animals withdrawn from the study because of disease progression. In order to account for patient attrition from progressive disease or unacceptable 
non-cardiac toxicity, more dogs per groups should be recruited. Thus, while the efficacy of any cardioprotective effect of dexrazoxane in tumourbearing dogs remains to be seen, the potential for such large prospective double-blinded trials is problematic because of issues of cost and practicality.

Similarly, it would be of questionable ethics to include a doxorubicin-alone control arm to a study of doxorubicin therapy in dogs with pre-existent heart disease, given that the cardioprotective effects of dexrazoxane in human patients are well established, ${ }^{10,51}$ as it is in healthy beagle dogs used in dexrazoxane preclinical studies. ${ }^{33}$ It is difficult to calculate the numbers of dogs that would be required to statistically validate cardioprotective effects in breeds of historically increased cardiomyopathic risk, given the uncertainty of rate of cardiomyopathy induction in such breeds treated with doxorubicin alone. In one study of dogs given doxorubicin at $30 \mathrm{mg} \mathrm{m}^{2}$ every 2 weeks for treatment of osteosarcoma, close to $50 \%$ of Doberman Pinschers (6 of 13) and Great Danes (3 of 7) in the study developed clinical cardiotoxicity. ${ }^{48}$ A power analysis suggests that to reduce the rate of cardiotoxicity in patients of these three breeds from 50 to $25 \%$, with alpha of $P<0.05$ and power of $80 \%, 55$ dogs in each of the doxorubicin and dox/dex groups would be required. A large-scale controlled prospective trial involving specific breeds of dogs may be invaluable in identifying those dogs at risk for cardiotoxicity (high-risk breeds) as well as others that may not traditionally be considered at risk. The potential benefit of dexrazoxane would be best assessed in such an at-risk cohort of dogs.

Based on human and veterinary clinical observations, it is possible that dogs with subclinical cardiomyopathies might benefit from dexrazoxane. In human medicine, additional markers to detect patients at higher risk of developing cardiotoxicity because of doxorubicin have been evaluated. Brain natriuretic peptide and troponin T levels, for example, have been studied as biomarkers of cardiotoxicity in rats, ${ }^{52}$ and it appears that patients at higher risk for developing cardiotoxicity may be identified before any echocardiographic changes are noted. ${ }^{51}$ Selting et al. ${ }^{53}$ evaluated cardiac troponin
I levels in dogs treated with doxorubicin for lymphoma and osteosarcoma. These investigators documented that cardiac troponin I levels were increased in advance of cardiac changes detectable either clinically or by post-treatment echocardiogram. Detection of subclinical cardiac damage through serum biomarkers may allow early application of dexrazoxane as a cardioprotectant in individual dogs at increased risk of doxorubicin cardiotoxicity. Moreover, endomyocardial biopsy is considered the 'gold standard' for detection of heart damage induced by anthracyclines. This invasive approach may not prove feasible or practical in a clinical setting. If risk of doxorubicin administration precludes its use, alternative chemotherapeutic drugs such as epirubicin or mitoxantrone may be used or changing to a different class of agents can be considered. Alterations in administration of doxorubicin may be another possible way of avoiding potential toxicity, such as administering doxorubicin as a constant rate infusion to reduce peak plasma concentrations. ${ }^{44}$

Ideally, in future studies, a doxorubicin-treated control group of breed- and age-matched dogs should be included to prospectively evaluate any cardiac benefit of dexrazoxane administered in conjunction with doxorubicin. Based on our assessment of the patient numbers required to complete such an effort, a multicenter trial with sufficient funding support would be necessary. Additional prospective studies may also address the ability of dexrazoxane administration to facilitate increase in doxorubicin dose or cumulative threshold escalation. Pharmacokinetic data of doxorubicin are also needed to identify kinetic changes that are noted with dox/dex administration which could affect potential toxicity, therapeutic efficacy and treatment protocol design. Dogs represent important translational pharmacokinetic and biologic models of drugs for human patients, based on similarities in pharmacokinetic parameters such as half-life, volume of distribution, metabolism and elimination. Because cardiomyopathic change is associated with doxorubicin administration to dogs in both investigational and clinical settings, it is reasonable to model cardioprotectant drugs in this species as well. $^{41,44,49}$ Other iron chelators and novel therapeutic drugs may be used in a clinical setting 
to ameliorate the cardiotoxicity of doxorubicin. In children treated with doxorubicin for various malignancies, the incidence of long-term medical problems such as heart disease and second malignancies may not be seen for months or even many years later. ${ }^{54}$ The application of cardioprotectant drugs such as dexrazoxane has been shown to be beneficial in human cancer patients and this is likely the case in dogs.

From the present study, it appears that one to two doses of dexrazoxane administered with doxorubicin are safe and well tolerated under the protocol parameters used here.

\section{Acknowledgments}

We would like to thank the oncology and cardiology technicians, and fellow oncology residents of MSU's Animal Cancer Care Clinic. Additional appreciation is given to Paul Curran at the MSU Center for Statistical Training and Consulting, and Robert Sanders, DVM, DACVIM (Cardiology) for evaluation of echocardiographic data. We have no conflicts of interest to report.

\section{References}

1. Lebrecht D, Geist A, Ketelson U-P, Haberstroh J and Setzer B, Walker UA. Dexrazoxane prevents doxorubicin-induced long-term cardiotoxicity and protects myocardial mitochondria from genetic and functional lesions in rats. British Journal of Pharmacology 2007; 151: 771-778.

2. Takemura G and Fujiwara H. Doxorubicin-induced cardiomyopathy from the cardiotoxic mechanisms to management. Progress in Cardiovascular Diseases 2007; 49: 330-352.

3. Gewirtz DA. A critical evaluation of the mechanisms of action proposed for the antitumor effects of the anthracycline antibiotics adriamycin and daunorubicin. Biochemical Pharmacology 1999; 57: 727-741.

4. Minotti G, Menna P, Salvatorelli E, Cairo G and Gianni L. Anthracyclines: molecular advances and pharmacologic developments in antitumor activity and cardiotoxicity. Pharmacological Reviews 2004; 56: $185-229$.

5. Hofland KF, Thougaard AV, Sehested M and Jensen PB. Dexrazoxane protects against myelosuppression from the DNA cleavage-enhancing drugs etoposide and daunorubicin but not doxorubicin. Clinical Cancer Research 2005; 10: 3915-3924.

6. Kellner U, Sehested M, Jensen PB, Gieseler F and Rudolph P. Culprit and victim - DNA topoisomerase II. The Lancet Oncology 2002; 3: 235-243.

7. Meresse P, Dechaux E, Monneret C and Bertounesque E. Etoposide: discovery and medicinal chemistry. Current Medicinal Chemistry 2004; 11: 2443-2466.

8. Nitiss JL and Beck WT. Antitopoisomerase drug action and resistance. European Journal of Cancer 1996; 32: 958-966.

9. Schuchter LM, Hensley ML, Meropol NJ and Winer EP. 2002 update of recommendations for the use of chemotherapy and radiotherapy protectants: clinical practice guidelines of the American Society of Clinical Oncology. Journal of Clinical Oncology 2002; 20: 2895-2903.

10. Swain SM and Vici P. The current and future role of dexrazoxane as a cardioprotectant in anthracycline treatment: expert panel review. Journal of Cancer Research and Clinical Oncology 2004; 130: 1-7.

11. Abeloff MD, Armitage JO, Niederhuber JE, Kastan MB and McKenna WG. Cardiac effects of cancer therapy. In: Clinical Oncology, 3rd edn., D Meloni, ed., Philadelphia, Churchill Livingstone, 2004: 1251-1263.

12. Von Hoff DD, Layard MW, Basa P, Davis HL, Von Hoff AL, Rozencweig M and Muggia FM. Risk factors for doxorubicin-induced congestive heart failure. Annals of Internal Medicine 1979; 91: 710-717.

13. Saini J, Rich MW and Lyss AP. Reversibility of severe left ventricular dysfunction due to doxorubicin cardiotoxicity: report of three cases. Annals of Internal Medicine 1987; 106: 814-816.

14. Dries DL, Strong MH, Cooper RS and Drazner MH. Efficacy of angiotensin-converting enzyme inhibition in reducing progression from asymptomatic left ventricular dysfunction to symptomatic heart failure in black and white patients. Journal of the American College of Cardiology 2002; 40: 311-317.

15. Postorino NC, Susaneck SJ, Withrow SJ, Macy DW and Harris C. Single agent therapy with adriamycin for canine lymphosarcoma. Journal of the American Animal Hospital Association 1989; 25: 221-225.

16. Maddison JE, Page SW and Church DB. Cancer chemotherapy. In: Small Animal Clinical Pharmacology, 1st ed., JE Maddison, SW Page and DB Church, eds., Philadelphia, WB Saunders, 2002: 293-326. 
17. Gianni L, Corden BJ and Myers CE. The biochemical basis of anthracycline toxicity and antitumor activity. In: Reviews in Biochemical Toxicology. E Hodgson, JR Bend and RM Philport, eds., Amsterdam, Elsevier, 1983: 1-82.

18. Gianni $\mathrm{L}$ and Myers CE. The role of free radical formation in the cardiotoxicity of anthracycline. In: Cancer Treatment and the Heart. FM Muggia, MD Green and JL Speyer, eds., Baltimore, MD, Johns Hopkins University Press, 1992: 9-46.

19. Doroshow JH. Effect of anthracycline antibiotics on oxygen radical formation in rat heart. Cancer Research 1983; 43: 460-472.

20. Doroshow JH, Locker GY and Myers CE. The enzymatic defenses of the mouse heart against reactive metabolites. The Journal of Clinical Investigation 1980; 65: 128-135.

21. Alderton PM, Gross J and Green MD. Comparative study of doxorubicin, mitoxantrone, and epirubicin in combination with ICRF-187 (ADR-529) in a chronic cardiotoxicity animal model. Cancer Research 1992; 52: 194-201.

22. Lebrecht D, Setzer B, Ketelsen UP, Haberstroh J and Walker UA. Time-dependent and tissue-specific accumulation of mtDNA and respiratory chain defects in chronic doxorubicin cardiomyopathy. Circulation 2003; 108: 2423-2429.

23. Lebrecht D, Kokkori A, Ketelsen UP, Setzer B and Walker UA. Tissue-specific mtDNA lesions and radical-associated mitochondrial dysfunction in human hearts exposed to doxorubicin. The Journal of Pathology 2005; 207: 436-444.

24. Kalyanaraman B, Perez-Reyes E and Mason RP. Spin-trapping and direct electron spin resonance investigations of the redox metabolism of quinone anticancer drugs. Biochimica et Biophysica Acta 1980; 630: 119-130.

25. Singal PK, Deally CM and Weinberg LE. Subcellular effects of adriamycin in the heart. A concise review. Journal of Molecular and Cellular Cardiology 1987; 19: $817-828$.

26. Myers C, Bonow R, Palmeri S, Jenkins J, Corden B, Locker G, Doroshow J and Epstein S. A randomized controlled trial assessing the prevention of doxorubicin cardiomyopathy by $\mathrm{N}$-acetylcysteine. Seminars in Oncology 1983; 10: 53-55.

27. Singal PK, Segstro RJ, Singh RP and Kutryk MJ. Changes in lysosomal morphology and enzyme activities during the development of adriamycin induced cardiomyopathy. The Canadian Journal of Cardiology 1985; 1: 139-147.

28. Odom AL, Hatwig CA, Stanley JS and Benson AM. Biochemical determinants of adriamycin toxicity in mouse liver, heart and intestine. Biochemical Pharmacology 1992; 43: 831-836.

29. Olson RD, MacDonald JS, vanBoxtel CJ, Boerth RC, Harbison RD, Slonim AE, Freeman RW and Oates JA. Regulatory role of glutathione and soluble sulfhydryl groups in the toxicity of adriamycin. Journal of Pharmacology and Experimental Therapeutics 1980; 215: 450-454.

30. Doroshow JH, Locker GY, Baldinger J and Myers CE. The effect of doxorubicin on hepatic and cardiac glutathione. Research Communications in Chemical Pathology and Pharmacology 1979; 26: 285-295.

31. Kane RC, McGuinn WD Jr, Daghe R, Justice R and Pazdur R. Dexrazoxane (TotectTM): FDA review and approval for the treatment of accidental extravasation following intravenous anthracycline chemotherapy. The Oncologist 2008; 13: 445-450.

32. Hasinoff BB. Chemistry of dexrazoxane and analogues. Seminars in Oncology 1998; 25: 3-9.

33. Hasinoff BB, Hellmann K, Herman EH and Ferrans VJ. Chemical, biological and clinical aspects of dexrazoxane and other bisdioxopiperazines. Current Medicinal Chemistry 1998; 5: 1-28.

34. Classen S, Olland S and Berger JM. Structure of the topoisomerase II ATPase region and its mechanism of inhibition by the chemotherapeutic agent ICRF-187. Proceedings of the National Academy of Sciences of the United States of America 2003; 100: 10629-10634.

35. Sehested M, Jensen PB, Sorensen BS, Holm B, Friche E and Demant EJ. Antagonistic effect of the cardioprotector (+)-1,2-bis(3,5-dioxopiperazinyl1-yl)propane (ICRF-187) on DNA breaks and cytotoxicity induced by the topoisomerase II directed drugs daunorubicin and etoposide (VP-16). Biochemical Pharmacology 1993; 46: 389-393.

36. Sehested M and Jensen PB. Mapping of DNA topoisomerase II poisons (etoposide, clerocidin) and catalytic inhibitors (aclarubicin, ICRF-187) to four distinct steps in the topoisomerase II catalytic cycle. Biochemical Pharmacology 1996; 51: 879-886.

37. Popelova O, Sterba M, Haskova P, Simunek T, Hroch M, Guncova I, Nachtigal P, Adamcova M, Gersl V and Mazurova Y. Dexrazoxane-afforded protection against chronic anthracycline cardiotoxicity in vivo: effective rescue of cardiomyocytes from apoptotic cell death. British Journal of Cancer 2009; 101: 792-802.

38. Sawyer DB, Fukazawa R, Arstall MA and Kelly RA. Daunorubicin-induced apoptosis in rat cardiac myocytes is inhibited by dexrazoxane. Circulation Research 1999; 84: 257-265. 
39. Konorev EA, Vanamala S and Kalyanaraman B. Differences in doxorubicin-induced apoptotic signaling in adult and immature cardiomyocytes. Free Radical Biology and Medicine 2008; 45: $1723-1728$.

40. Lyu YL, Kerrigan JE, Lin CP, Azarova AM, Tsai YC, Ban Y and Liu LF. Topisomerase II $\beta$-mediated DNA double-strand breaks: implications in doxorubicin cardiotoxicity and prevention by dexrazoxane. Cancer Research 2007; 67: 8839-8846.

41. Baldwin JR, Phillips BA, Overmyer SK, Hatfield NZ and Narang PK. Influence of the cardioprotective agent dexrazoxane on doxorubicin pharmacokinetics in the dog. Cancer Chemotherapy and Pharmacology 1992; 30: 433-438.

42. Vail, DM. Veterinary Co-operative Oncology Group - common terminology criteria for adverse events (VCOG-CTCAE) following chemotherapy or biological antineoplastic therapy in dogs and cats v1.0. Journal of Veterinary and Comparative Oncology 2004; 2: 194-213.

43. Mutsaers AJ, Glickman NW, DeNicola DB, Widmer WR, Bonney PL, Hahn KA and Knapp DW. Evaluation of treatment with doxorubicin and piroxicam or doxorubicin alone for multicentric lymphoma in dogs. Journal of the American Veterinary Medical Association 2002; 220: 1813-1817.

44. Gillings SL, Johnson J, Fulmer A and Hauck ML. Effect of a 1-hour IV infusion of doxorubicin on the development of cardiotoxicity in dogs as evaluated by electrocardiography and echocardiography. Veterinary Therapeutics 2009; 10: 46-58.

45. Sorenmo, KU, Baez JL, Clifford CA, Mauldin E, Overley B, Skorupski K, Bachman R, Samluk M and Shofer F. Efficacy and toxicity of a dose-intensified doxorubicin protocol in canine hemangiosarcoma. Journal of Veterinary Internal Medicine 2004; 18: 209-213.

46. Sparreboom A, Wolff AC, Mathijssen RHJ, Chatelut E, Rowinsky EK, Verweij J and Baker SD. Evaluation of alternate size descriptors for dose calculation of anticancer drugs in the obese. Journal of Clinical Oncology 2007; 25: 4707-4713.

47. Singal PK and Iliskovic N. Doxorubicin-induced cardiomyopathy. New England Journal of Medicine 1998; 339: 900-905.
48. Moore AS, Dernell WS, Ogilvie GK, Kristal O, Elmslie R, Kitchell B, Susaneck S, Rosenthal R, Klein MK, Obradovich J, Legendre A, Haddad T, Hahn K, Powers BA and Warren D. Doxorubicin and BAY 12-9566 for the treatment of osteosarcoma in dogs: a randomized, double-blind, placebo-controlled study. Journal of Veterinary Internal Medicine 2007; 21: 783-790.

49. Mauldin GE, Fox PR, Patnaik AK, Bond BR, Mooney SC and Matus RE. Doxorubicin-induced cardiotoxicosis: clinical features in 32 dogs. Journal of Veterinary Internal Medicine 1992; 2: 82-88.

50. Page RL, Macy DW, Ogilvie GK, Rosner GL, Dewhirst MW, Thrall DE, Withrow SJ, McEntee MC, Cline JM, Heidner GL, Novotney CA and Gillette EL. Phase III evaluation of doxorubicin and whole-body hyperthermia in dogs with lymphoma. International Journal of Hyperthermia 1992; 8: 187-197.

51. Lipshultz SE, Rifai N, Dalton VM, Levy DE, Silverman LB, Lipsitz SR, Colan SD, Asselin BL, Barr RD, Clavell LA, Hurwitz CA, Moghrabi A, Samson Y, Schorin MA, Gelber RD and Sallan SE. The effect of dexrazoxane on myocardial injury in doxorubicin-treated children with acute lymphoblastic leukemia. New England Journal of Medicine 2004; 351: 145-153.

52. Herman EH, Lipschultz, SE, Rifai N, Zhang J, Papoian T, Yu Z, Takeda K and Ferrans VJ. Use of cardiac troponin-t levels as an indicator of doxorubicin-induced cardiotoxicity. Cancer Research 1998; 58: 195-197.

53. Selting KA, Lana SE, Ogilvie GK, Olmstead A, Mykles DL, Bright J, Richardson KL, Walton JA, Monnet E and Fettman MJ. Cardiac troponin I in canine patients with lymphoma and osteosarcoma receiving doxorubicin: Comparison with clinical heart disease in a retrospective analysis. Journal of Veterinary and Comparative Oncology 2004; 2: 142-156.

54. Oeffinger KC, Mertens AC, Sklar CA, Kawashima T, Hudson MH, Meadows AT, Friedman DL, Marina N, Hobbie W, Kadan-Lottick NS, Schwartz CL, Leisenring W and Robison LL. Chronic health conditions in adult survivors of childhood cancer. New England Journal of Medicine 2006; 355: 1572-1582. 